\title{
IMPROVING THE EFFICIENCY OF PROBIT ESTIMATORS
}

\author{
Andrew Chesher*
}

\begin{abstract}
The efficiency with which coefficients in probit models are estimated is improved by exploiting data on continuous ancillary variates. In this paper the resulting gains in efficiency are examined and illustrative calculations are provided. Extra precision is achieved at the cost of making an extra assumption but this assumption can be tested. It is shown that fully efficient maximum likelihood estimation of the probit model with a continuous ancillary variate can be achieved by a simple two step procedure involving an ordinary least squares and a probit estimation.
\end{abstract}

Received for publication March 23, 1983. Revision accepted for publication October 18, 1983.

* University of Bristol.

I am grateful to Joanna Gomulka and Tony Lancaster for helpful comments. Errors are my responsibility. The work reported here is part of a project on the Microeconometrics of Labour Market Transitions supported by the Social Science Research Council.

\section{Introduction}

Probit analysis of binary data is now widely practised by social scientists. Sometimes data are available on endogenous continuous variates which, given exogenous variables, are correlated with the binary variate on which probit analysis is performed. For example, when estimating models for housing tenure choice, household income or expenditure on some nondurable goods may be available. And when estimating a model for the return to work of an unemployed worker in some time interval, tenure of or wage in the previous job may be available. This paper provides a simple computational procedure for using ancillary data of this sort efficiently and a method for obtaining estimates of the asymptotic variances of the resulting estimators. Finally the gain in 
efficiency obtained by using the ancillary data is investigated.

Suppose that, given a vector $x$, two variates $y_{1}$ and $y_{2}$ are bivariate normally distributed:

$$
\left[\begin{array}{l|l}
y_{1} & x \\
y_{2} &
\end{array}\right] \sim N_{2}\left[\left[\begin{array}{c}
x^{\prime} \pi_{1} \\
x^{\prime} \pi_{2}
\end{array}\right],\left[\begin{array}{cc}
\sigma_{11} & \rho \sigma_{11}^{1 / 2} \\
\rho \sigma_{11}^{1 / 2} & 1
\end{array}\right]\right] \text {. }
$$

Realisations of $y_{1}$ are observed but $y_{2}$ is not observable. Instead realisations of a binary variate $D$ are obtained $^{1}$ where $D=1$ if $y_{2} \geq 0, D=0$ if $y_{2}<0$. This model provides a framework for the analysis of binary data when jointly dependent continuous variates are available and it can be regarded as the reduced form of a simultaneous equations model in binary and continuous variates (see Heckman (1978)). The model also finds application in the sample selection bias area if we attach to it a variate $y_{3}$ only observed when $D=1$. Heckman's (1979) two-step procedure uses an estimate of $\pi_{2}$ to calculate the normal hazard function included as an extra "regressor" in the $y_{3}$ equation. In all these contexts more efficient estimation of $\pi_{2}$ is of interest.

With $y_{2}$ observed (1) can be written as a pair of seemingly unrelated regression equations with identical regressors and separate ordinary least squares (OLS) estimation of the two equations is efficient. Data on $y_{1}$ are not informative about $\pi_{2}$ when $y_{2}$ is observed (see Conniffe (1982)). The coarse grouping of $y_{2}$ into the two classes indicated by $D$ destroys information but the ordinary least squares (OLS) estimators of $\pi_{1}$ and $\sigma_{11}$ can still be calculated and are therefore still efficient. Since the data are informative about the conditional correlation of $y_{1}$ and $y_{2}$ given $x$, the magnitude of $y_{1}$ is generally informative about the location of $y_{2}$ within the two classes $\left(y_{2} \geq 0, y_{2}<0\right)$ into which it is coded. Consequently, with $D$ observed in place of $y_{2}$, the "single equation" probit estimator of $\pi_{2}$ which ignores the $y_{1}$ data, using just $D$ and $x$ data, is generally inefficient.

It is shown in section II that the fully efficient maximum likelihood (ML) estimator of $\pi_{2}$ is obtained by a probit analysis of $D$ on $x$ and $y_{1}$ so that ML estimation of (1) can be achieved using standard OLS and probit analysis software with negligible increase in computational cost over separate analysis of the $y_{1}$ and $D$ data. In section III the magnitude of the (asymptotic) efficiency gain, which is nonzero for $\rho \neq 0$, is considered.

\section{Marginal and Joint Maximum Likelihood Estimators}

From the marginal distributions of $y_{1}$ and $D$ the $\log$ likelihood functions (2) and (3) are obtained, assuming

\footnotetext{
${ }^{1}$ Since $y_{2}$ is not observable the normalisation $\operatorname{var}\left(y_{2} \mid x\right)=1$ is innocuous.
}

$n$ independent realisations of $y_{1}$ and $D$ given $x$.

$$
\begin{aligned}
& L_{m}\left(\pi_{1}, \sigma_{11}\right)=-\frac{n}{2} \log 2 \pi-\frac{n}{2} \log \sigma_{11} \\
&-\frac{1}{2 \sigma_{11}} \sum\left(y_{1 i}-x_{i}^{\prime} \pi_{1}\right)^{2} \\
& L_{m}\left(\pi_{2}\right)=\sum \log \bar{\Phi}\left(\left(1-2 D_{i}\right) x_{i}^{\prime} \pi_{2}\right) .
\end{aligned}
$$

Here $\bar{\Phi}$ is the complement of the standard normal distribution function. All summations here and later are over $i=1$ to $n$.

The maximum likelihood estimators from (2) and (3) are $\hat{\pi}_{1}$, the OLS estimator given by regressing $y_{1}$ on $x$, $\hat{\sigma}_{11}$, the mean squared OLS residuals from this regression and $\hat{\pi}_{2}$, the probit estimator obtained from probit analysis of $D$ using $x$ as explanatory variables. These estimators, which are called marginal maximum likelihood (MML) estimators, have the usual optimality properties under the assumption that $y_{1}$ given $x$ and $y_{2}$ given $x$ are marginally normally distributed.

If $y_{1}$ and $y_{2}$ are assumed to be jointly normally distributed given $x$ then the joint log-likelihood is

$$
\begin{aligned}
& L_{J}\left(\gamma_{1}, \theta_{1}, \gamma_{2}, \theta_{2}\right) \\
& =\sum \log \bar{\Phi}\left(\left(1-2 D_{i}\right)\left(x_{i}^{\prime} \gamma_{2}+\theta_{2} y_{1 i}\right)\right) \\
& \quad-\frac{n}{2} \log 2 \pi-\frac{n}{2} \log \theta_{1}-\frac{1}{2 \theta_{1}} \sum\left(y_{1 i}-x_{i}^{\prime} \gamma_{1}\right)^{2} .
\end{aligned}
$$

The log likelihood (4) is written using the decomposition $P\left(y_{1} \cap D \mid x\right)=P\left(D \mid y_{1} \cap x\right) P\left(y_{1} \mid x\right)$ and the parameterisation:

$$
\begin{aligned}
& \gamma_{1}=\pi_{1} \\
& \theta_{1}=\sigma_{11} \\
& \gamma_{2}=\left(1-\rho^{2}\right)^{-1 / 2}\left(\pi_{2}-\rho \sigma_{11}^{-1 / 2} \pi_{1}\right) \\
& \theta_{2}=\rho \sigma_{11}^{-1 / 2}\left(1-\rho^{2}\right)^{-1 / 2} .
\end{aligned}
$$

Setting the first derivatives of (4) equal to zero it can be seen that the joint maximum likelihood (JML) estimators of $\gamma_{1}\left(=\pi_{1}\right)$ and $\theta_{1}\left(=\sigma_{11}\right)$ are identical to the MML estimators ${ }^{2}$ of $\pi_{1}$ and $\sigma_{11}$ and that the JML estimators of $\gamma_{2}$ and $\theta_{2}$ are obtained by a simple probit analysis of the $D$ data using $x$ and $y_{1}$ as explanatory variables.

The inverse of the transformation (5) is

$$
\begin{aligned}
\pi_{1} & =\gamma_{1} \\
\sigma_{11} & =\theta_{1} \\
\pi_{2} & =\left(1+\theta_{1} \theta_{2}^{2}\right)^{-1 / 2}\left(\gamma_{2}+\theta_{2} \gamma_{1}\right) \\
\rho & =\theta_{1}^{1 / 2} \theta_{2}\left(1+\theta_{1} \theta_{2}^{2}\right)^{-1 / 2}
\end{aligned}
$$

which enables unique estimates of $\pi_{1}, \pi_{2}, \sigma_{11}$ and $\rho$ to be obtained from estimates of $\gamma_{1}, \gamma_{2}, \theta_{1}$ and $\theta_{2}$. The invariance properties of maximum likelihood estimators

\footnotetext{
${ }^{2}$ This is also true when elements of $\pi_{1}$ are constrained to be zero.
} 
ensure that the resulting estimators $\left(\tilde{\pi}_{1}, \tilde{\pi}_{2}, \tilde{\sigma}_{11}, \tilde{\rho}\right)$ possess the usual optimality properties.

The preceding argument demonstrates that full maximum likelihood estimation of the parameters of (1) can be achieved via a simple two-step procedure comprising OLS estimation of $\pi_{1}$ and $\sigma_{11}$ using the $y_{1}$ and $x$ data and probit analysis of the $D, x$ and $y_{1}$ data. If $M$ continuous variates $y_{1}$ are available then the probit analysis uses $x$ and the vector $y_{1}$ as explanatory variables. This two-step procedure is essentially the reverse of that suggested by Heckman (1979) to correct for sample selection bias, as is expected given the decomposition of $P\left(y_{1} \cap D \mid x\right)$ used in writing down the joint log likelihood (4).

So, joint maximum likelihood estimation is computationally straightforward. But what gains in efficiency can be expected from exploiting ancillary continuous data? This question is investigated in the next section.

\section{Asymptotic Variances and Relative Efficiency}

First consider the marginal maximum likelihood estimator, $\hat{\pi}_{2}$. The asymptotic variance matrix of $\sqrt{n}\left(\hat{\pi}_{2}\right.$ $\left.-\pi_{2}\right), \overline{\operatorname{var}}\left(\hat{\pi}_{2}\right)$ is:

$$
\overline{\operatorname{var}}\left(\hat{\pi}_{2}\right)=\left\{\operatorname{plim} n^{-1} \sum_{i=1}^{n} h\left(x_{i}^{\prime} \pi_{2}\right) h\left(-x_{i}^{\prime} \pi_{2}\right) x_{i} x_{i}^{\prime}\right\}^{-1}
$$

where $h(\quad)$ is the standard normal hazard function. It is assumed that probability limits exist and where appropriate are non-singular.

If $\phi(w)$ and $\Phi(w)$ are the standard normal density and distribution functions then $h(w) h(-w)=$ $\phi(w)^{2} / \Phi(w) \Phi(-w)$ which attains its maximum of $2 / \pi$ $=0.637$ when $w=0$. With $y_{2}$ observed, the asymptotic replaced by $\operatorname{var}\left(y_{2} \mid x_{i}\right)^{-1}=1.0$. So the grouping of $y_{2}$ into two classes coded by $D$ results in an increase in the asymptotic variance of $\pi_{2}$ of at least $100\left((.637)^{-1}-\right.$ $1) \%=57 \%$. Assuming joint normality of $y_{1}$ and $y_{2}$ given $x$ and utilising the $y_{1}$ data as described in the previous section allows some of this lost efficiency to be regained but the $y_{1}$ data cannot be used to reduce the variance of $\tilde{\pi}_{2}$ below that attained when $y_{2}$ is observed

Now consider the asymptotic variance matrix of the JML estimator. Since the $\log$ likelihood (4) is an additively separable function of $\left(\gamma_{1}, \theta_{1}\right)$ and $\left(\gamma_{2}, \theta_{2}\right)$ the asymptotic covariance matrix of the JML estimator of $\left(\gamma_{1}, \theta_{1}, \gamma_{2}, \theta_{2}\right)$ is block diagonal given by

$$
\overline{\operatorname{var}}\left[\begin{array}{l}
\tilde{\gamma}_{1} \\
\tilde{\theta}_{1} \\
\tilde{\gamma}_{2} \\
\tilde{\theta}_{2}
\end{array}\right]=V=\left[\begin{array}{cc:cc}
\theta_{1} Q^{-1} & 0 & 0 & 0 \\
0 & 2 \theta_{1}^{2} & 0 & 0 \\
\hdashline 0 & 0 & & \\
0 & 0 & & V_{2}
\end{array}\right]
$$

where

$$
\begin{aligned}
& V_{2}^{-1}=\operatorname{plim} n^{-1} \sum E h\left(w_{i}\right) h\left(-w_{i}\right) \\
& \times\left[\begin{array}{c}
x_{i} \\
\hdashline \frac{w_{i}-x_{i}^{\prime} \gamma_{2}}{\theta_{2}}
\end{array}\right]\left[\begin{array}{l:l} 
& w_{i}^{\prime}-x_{i}^{\prime} \gamma_{2} \\
x_{i} & \theta_{2}
\end{array}\right],
\end{aligned}
$$

expectation is with respect to $w_{i}$ given $x_{i}$ which is

$$
\left.N_{1}\left[x_{i}^{\prime}\left(\gamma_{2}+\theta_{2} \gamma_{1}\right), \theta_{2}^{2} \theta_{1}\right)\right]
$$

and

$$
Q=\operatorname{plim} n^{-1} \sum_{i=1}^{n} x_{i} x_{i}^{\prime}
$$

To obtain the asymptotic variance matrix of the JML estimators of $\left(\pi_{1}, \sigma_{11}, \pi_{2}, \rho\right)$, consider the Jacobian, $\Delta$, of the transformation (5):

$$
\begin{aligned}
& \Delta=\left[\begin{array}{cc|cc}
I_{m} & 0 & 0 & 0 \\
0 & 1 & 0 & 0 \\
--\frac{\rho}{\sigma_{11}^{1 / 2}} I_{m} & \frac{-\rho^{2} \pi_{2}}{2 \sigma_{11}} & \left(1-\rho^{2}\right)^{1 / 2} I_{m} & \left(1-\rho^{2}\right)^{1 / 2}\left(\pi_{1}-\sigma_{11}^{1 / 2} \rho \pi_{2}\right) \\
0 & \frac{\rho}{2 \sigma_{11}}\left(1-\rho^{2}\right) & 0 & \sigma_{11}^{1 / 2}\left(1-\rho^{2}\right)^{3 / 2}
\end{array}\right] \\
& =\left[\begin{array}{c:c}
I_{m+1} & 0 \\
\hdashline A & B
\end{array}\right], \quad \text { say. }
\end{aligned}
$$

variance of the ML estimator of $\pi_{2}$ (the OLS estimator from regressing $y_{2}$ on $\left.x\right)$ is (7) with $h\left(x_{i}^{\prime} \pi_{2}\right) h\left(-x_{i}^{\prime} \pi_{2}\right)$
A standard argument exploiting the local linearity of the transformation (5) gives, for the asymptotic variance 
matrix of $\left(\tilde{\pi}_{1}, \tilde{\sigma}_{11}, \tilde{\pi}_{2}, \tilde{\rho}\right), \Delta V \Delta^{\prime}$, which is

$$
\overline{\operatorname{var}}\left[\begin{array}{c}
\tilde{\pi}_{1} \\
\tilde{\sigma}_{11} \\
\tilde{\pi}_{2} \\
\tilde{\rho}
\end{array}\right]=\left[\begin{array}{cc|cc}
\sigma_{11} Q^{-1} & 0 & \rho \sigma_{11}^{1 / 2} Q_{2}^{-1} & 0 \\
0 & 2 \sigma_{11}^{2} & -\rho^{2} \sigma_{11} \pi_{2}^{\prime} & \sigma_{11} \rho\left(1-\rho^{2}\right) \\
-0 \sigma_{11}^{1 / 2} Q^{-1} & -\rho^{2} \sigma_{11} \pi_{2} \\
0 & \sigma_{11} \rho\left(1-\rho^{2}\right) & {\left[\begin{array}{cc}
\rho^{2} Q^{-1}+\frac{\rho^{4}}{2} \pi_{2} \pi_{2}^{\prime} & \frac{-\rho^{3}\left(1-\rho^{2}\right) \pi_{2}}{2} \\
\frac{-\rho^{3}\left(1-\rho^{2}\right) \pi_{2}^{\prime}}{2} & \frac{\rho^{2}\left(1-\rho^{2}\right)^{2}}{2}
\end{array}\right]+B V_{2} B^{\prime}}
\end{array}\right] .
$$

Some tedious algebra shows that $\overline{\operatorname{var}}\left(\hat{\pi}_{2}\right)$ exceeds $\overline{\operatorname{var}}\left(\tilde{\pi}_{2}\right)$ by a positive semidefinite matrix which converges to zero as $\rho$ passes to zero. ${ }^{3}$

The gain in efficiency from using the $y_{1}$ data is obtained at the cost of making an extra assumption, namely, that $y_{1}$ and $y_{2}$ given $x$ are jointly as well as marginally normally distributed. When this additional assumption is incorrect the JMLE $\tilde{\pi}_{2}$ may be inconsistent but while the marginal normality assumption is correct $\hat{\pi}_{2}$, the MMLE, is consistent. When the joint normality assumption is correct the JMLE is efficient relative to the MMLE. So $\tilde{\pi}_{2}-\hat{\pi}_{2}$ provides the basis for a Hausman (1978) test of the additional assumption.

The variance matrix (10) can be estimated in the two-step procedure outlined earlier. $Q^{-1}$ is consistently estimated by $\left(n^{-1} \sum x_{i} x_{i}^{\prime}\right)^{-1}$ and $\pi_{1}, \pi_{2}, \sigma_{11}, \rho$ by their JML estimators. The matrix $V_{2}$ is consistently estimated by the inverse of $n^{-1}$ times the Hessian of the "log likelihood" used in the probit analysis of $D$ on $y_{1}$ and $x$. Thus all the required elements of $\overline{\operatorname{var}}\left(\tilde{\pi}_{2}\right)$ are readily available as normal outputs of the OLS and probit programmes used in the two-step procedure.

Now consider the magnitude of the efficiency gain obtained by using the ancillary continuous data. When there is no regression, so that $x$ is scalar and equal to one, it is possible to calculate this gain for most interesting values of $\pi_{2}$ and $\rho$. The results are shown in table 1 where two variances $\overline{\operatorname{var}}\left(\tilde{\pi}_{2} \mid \rho\right)$ and $\overline{\operatorname{var}}\left(\tilde{\pi}_{2}\right)$ are given, since in this model the asymptotic variance of $\tilde{\pi}_{2}$ depends on whether $\rho$ is known or estimated. The column headed $\rho^{2}=0$ gives $\overline{\operatorname{var}}\left(\hat{\pi}_{2}\right)$. The three variances are invariant under changes in the signs of $\pi_{2}$ and $\rho$ and are smallest when $\pi_{2}=0$, i.e., when $P[D=1]=0.5$. In this "no regression" case, $\hat{\pi}_{2}=-\bar{\Phi}^{-1}\left(n_{1} / n\right)$ where $n_{1}$ is the number of observations with $D=1$. Though $\operatorname{var}\left(n_{1} / n\right)$ is at a maximum when $\pi_{2}=0$, the increasing flatness of the $\bar{\Phi}^{-1}$ function results in $\overline{\operatorname{var}}\left(\hat{\pi}_{2}\right)$ being at a minimum when $\pi_{2}=0$. For all $\rho^{2}<1, \overline{\operatorname{var}}\left(\hat{\pi}_{2}\right)$ $\geq \overline{\operatorname{var}}\left(\tilde{\pi}_{2}\right) \geq \overline{\operatorname{var}}\left(\tilde{\pi}_{2} \mid \rho\right)>\overline{\operatorname{var}}\left(\hat{\pi}_{2} \mid y_{2}\right.$ observed $)=1$, with equalities holding when $\rho=0$. The greatest gains in

\footnotetext{
${ }^{3}$ When $\rho^{2}=1$ and $\rho$ is known, $y_{2}$ can be "reconstructed" from $y_{1}$ and $x$ and all the lost efficiency due to grouping $y_{2}$ can be regained. It seems plausible that this is also true, asymptotically, when $\rho$ is unknown and in fact $\rho^{2}=1$.
}

efficiency are obtained for large $\left|\pi_{2}\right|$, when the event $D=1$ is either relatively rare or relatively common, and for large $\rho^{2}$.

Now suppose there is regression and write $x^{\prime} \pi_{2}=\pi_{20}$ $+\pi_{21} x_{1}+\pi_{22} x_{2}$ where $x_{1}$ and $x_{2}$ are $N(0,1)$, $\operatorname{cor}\left(x_{1}, x_{2}\right)=r$ and realisations of $\left(x_{1} x_{2}\right)$ are independent. Taking expectations over $x$ gives expressions for $\overline{\operatorname{var}}\left(\hat{\pi}_{2 i}\right)$ and $\overline{\operatorname{var}}\left(\tilde{\pi}_{2 i} \mid \rho\right)$ which are evaluated (with $\pi_{21}=$ $\pi_{22}$ denoted by $\pi_{2 .}$.) to produce table 2 . Only one variance is reported since under these conditions variances of the estimators of $\pi_{21}$ and $\pi_{22}$ are equal. Asymptotic covariances, which are not reported, are reduced in magnitude on introducing the $y_{1}$ data by approximately the same amount as is the asymptotic variance.

The asymptotic variance of the MML estimator $\hat{\pi}_{2 i}$ (see the columns headed $\rho^{2}=0$ ) increases with $\pi_{20}$ and $\pi_{2}$. With $\pi_{2 .}=0$ minimum asymptotic variance is obtained when $x_{1}$ and $x_{2}$ are uncorrelated but as $\pi_{2}$ increases through positive values the value of $r$ for which the asymptotic variance is at a minimum declines. Comparing the entries in table 2 with the last column of table 2, which gives $\overline{\operatorname{var}}\left(\hat{\pi}_{2 i} \mid y_{2}\right.$ observed $)=\left(1-r^{2}\right)^{-1}$, it can be seen that the loss in efficiency due to the grouping of $y_{2}$ into the two classes indicated by $D$ increases with $\pi_{20}$ and with $\pi_{2}$ and for non-zero $\pi_{2}$. varies with $r$, generally increasing as the magnitude of $r$ increases.

TABle 1. - Asymptotic VARIANCes of $\tilde{\pi}_{2}, \rho$ KNOWN (UPPER ENTRIES), $\rho$ ESTIMATED (LOWER ENTRIES)

\begin{tabular}{lllll}
\hline \hline & \multicolumn{5}{c}{$\rho^{2}$} \\
\cline { 2 - 5 }$\pi_{2}$ & $0.0^{\mathrm{a}}$ & 0.4 & 0.8 & 0.95 \\
\hline 0 & 1.57 & 1.55 & 1.43 & 1.26 \\
& 1.57 & 1.55 & 1.43 & 1.26 \\
\hline 1.0 & 2.29 & 2.17 & 1.82 & 1.46 \\
& 2.29 & 2.27 & 2.15 & 1.91 \\
\hline 2.0 & 7.62 & 7.00 & 5.10 & 3.21 \\
& 7.62 & 7.52 & 6.48 & 5.03 \\
\hline 2.5 & 20.09 & 18.58 & 13.46 & 7.81 \\
& 20.09 & 19.71 & 15.89 & 10.71 \\
\hline
\end{tabular}

${ }^{\mathrm{a}}$ When $\rho^{2}=0$, entries give $\overline{\operatorname{var}}\left(\hat{\pi}_{2}\right)=\overline{\operatorname{var}}\left(\tilde{\pi}_{2}\right)$. 
TABle 2. - Asymptotic VARIANCES OF $\tilde{\pi}_{2,}, \rho$ KNOWN, WheRE $x^{\prime} \pi_{2}=\pi_{20}+\pi_{2 .} x+\pi_{2 .} x_{2}, r=\operatorname{cor}\left(x_{1}, x_{2}\right)$

\begin{tabular}{|c|c|c|c|c|c|c|c|c|c|c|}
\hline \multirow[b]{2}{*}{$\pi_{2}$} & \multirow[b]{2}{*}{$r$} & \multicolumn{4}{|c|}{$\pi_{20}=0.0$} & \multicolumn{4}{|c|}{$\pi_{20}=2.0$} & \multirow{2}{*}{$\begin{array}{c}\overline{\operatorname{var}}\left(\hat{\pi}_{2 .} \mid\right. \\
\left.y_{2} \text { observed }\right)\end{array}$} \\
\hline & & $0^{\mathrm{a}}$ & 0.4 & 0.8 & 0.95 & $0^{\mathrm{a}}$ & 0.4 & 0.8 & 0.95 & \\
\hline \multirow{7}{*}{0} & -.9 & 8.27 & 8.18 & 7.55 & 6.64 & 40.1 & 36.8 & 26.8 & 16.9 & 5.26 \\
\hline & -.6 & 2.45 & 2.43 & 2.24 & 1.97 & 11.9 & 10.9 & 8.0 & 5.0 & 1.56 \\
\hline & -.3 & 1.73 & 1.71 & 1.58 & 1.39 & 8.4 & 7.7 & 5.6 & 3.5 & 1.10 \\
\hline & 0.0 & 1.57 & 1.55 & 1.43 & 1.26 & 7.6 & 7.0 & 5.1 & 3.2 & 1.00 \\
\hline & .3 & 1.73 & 1.71 & 1.58 & 1.39 & 8.4 & 7.7 & 5.6 & 3.5 & 1.10 \\
\hline & 6 & 2.45 & 2.43 & 2.24 & 1.97 & 11.9 & 10.9 & 8.0 & 5.0 & 1.56 \\
\hline & .9 & 8.27 & 8.18 & 7.55 & 6.64 & 40.1 & 36.8 & 26.8 & 16.9 & 5.26 \\
\hline \multirow{7}{*}{1} & -.9 & 10.11 & 9.74 & 8.52 & 7.13 & 40.8 & 36.2 & 25.7 & 16.1 & 5.26 \\
\hline & -.6 & 4.65 & 4.26 & 3.37 & 2.55 & 12.8 & 10.9 & 7.5 & 4.7 & 1.56 \\
\hline & -.3 & 4.25 & 3.81 & 2.87 & 2.05 & 9.3 & 7.9 & 5.4 & 3.3 & 1.10 \\
\hline & 0.0 & 4.48 & 3.97 & 2.92 & 2.02 & 8.4 & 7.2 & 4.9 & 3.0 & 1.00 \\
\hline & .3 & 5.16 & 4.54 & 3.31 & 2.28 & 8.8 & 7.5 & 5.1 & 3.2 & 1.10 \\
\hline & .6 & 6.91 & 6.08 & 4.47 & 3.11 & 10.9 & 9.3 & 6.4 & 4.1 & 1.56 \\
\hline & .9 & 18.89 & 16.77 & 12.71 & 9.26 & 28.1 & 24.1 & 17.1 & 11.4 & 5.26 \\
\hline \multirow{7}{*}{2} & -.9 & 16.62 & 15.18 & 11.87 & 8.85 & 45.9 & 39.3 & 26.9 & 16.6 & 5.26 \\
\hline & -.6 & 13.93 & 11.96 & 8.14 & 5.00 & 22.1 & 18.7 & 12.3 & 7.1 & 1.56 \\
\hline & -.3 & 15.71 & 13.31 & 8.76 & 5.08 & 21.2 & 17.8 & 11.5 & 6.5 & 1.10 \\
\hline & 0.0 & 17.89 & 15.08 & 9.81 & 5.56 & 22.3 & 18.7 & 12.0 & 6.7 & 1.00 \\
\hline & .3 & 20.58 & 17.30 & 11.22 & 6.34 & 24.5 & 20.5 & 13.2 & 7.4 & 1.10 \\
\hline & .6 & 25.03 & 21.03 & 13.70 & 7.84 & 28.9 & 24.2 & 15.6 & 8.8 & 1.56 \\
\hline & .9 & 47.92 & 40.28 & 26.95 & 16.52 & 54.1 & 45.3 & 29.9 & 18.0 & 5.26 \\
\hline
\end{tabular}

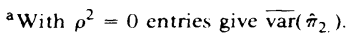

The gain in efficiency on introducing the $y_{1}$ dat? increases with $\rho^{2}, \pi_{20}$ and $\pi_{2}$ and varies with $r=$ $\operatorname{cor}\left(x_{1}, x_{2}\right)$. For models in which the exogenous variables are highly correlated and for which the event $D=1$ is relatively rare or relatively common, considerable gains in efficiency can be achieved by using the joint maximum likelihood estimator which, as noted earlier, is simple to calculate.

\section{REFERENCES}

Conniffe, Denis, "Testing the Assumptions of Seemingly Unrelated Regressions," this REVIEW 64 (Feb. 1982), 172-174. Hausman, Jerry A., "Specification Tests in Econometrics," Econometrica 46 (Nov. 1978), 1251-1271.

Heckman, James, "Dummy Endogenous Variables in a Simultaneous Equation System,” Econometrica 46 (July 1978), 931-959.

, "Sample Selection Bias as Specification Error," Econometrica 47 (Jan. 1979), 153-161. 